\title{
Acknowledgment to Reviewers of Galaxies in 2020
}

\section{Galaxies Editorial Office}

Citation: Galaxies Editorial Office. Acknowledgment to Reviewers of Galaxies in 2020. Galaxies 2021, 9, 8. https://doi.org/10.3390/

galaxies 9010008

Published: 25 January 2021

Publisher's Note: MDPI stays neutral with regard to jurisdictional claims in published maps and institutional affiliations.

Copyright: (c) 2021 by the author. Licensee MDPI, Basel, Switzerland. This article is an open access article distributed under the terms and conditions of the Creative Commons Attribution (CC BY) license (http://creativecommons.org/licenses /by/4.0/).

MDPI AG, St. Alban-Anlage 66, 4052 Basel, Switzerland

Peer review is the driving force of journal development, and reviewers are gatekeepers who ensure that Galaxies maintains its standards for the high quality of its published papers. Thanks to the cooperation of our reviewers, in 2020, the median time to first decision was 18 days and the median time to publication was 46 days. The editors would like to express their sincere gratitude to the following reviewers for their precious time and dedication, regardless of whether the papers were finally published:

Abdujabbarov, Ahmadjon

Acedo, Luis

Aleman, Isabel

Alexandros, Chiotellis

Balick, Bruce

Bargueno, Pedro

Barría, Daniela

Bassan, Massimo

Beaklini, Pedro P. B.

Beck, Geoff

Benbow, Wystan

Berg, Danielle

Bhatta, Gopal

Bhatti, Muhammad Zaeem Ul Haq

Biermann, Peter

Birnholtz, Ofek

Blackman, Eric

Boissay-Malaquin, Rozenn

Boumis, Panayotis

Bozorgnia, Nassim

Brack, Andre

Brandenburg, Axel

Brown, Daniel D.

Bulik, Tomasz

Cai, Yi-Fu

Cao, Xinwu

Capocasa, Eleonora

Carini, Mike

Carpano, Stefania

Casagrande, Luca

Cembranos, Jose

Chakraborty, Nachiketa

Chamandy, Luke

Chan, Man Ho

Chang, Chung-Ping
Charles, Eric

Chen, Long

Cheng, Cheng

Chisholm, John

Christodoulou, Dimitris

Chu, You-Hua

Claudi, Riccardo

Conroy, Kyle

Corradi, Romano

Criss, Robert E.

Crowther, Paul

Cruden, Brett A.

D'Ammando, Filippo

Da Silva, Mario Lino

Danilishin, Stefan

De Cesare, Marco

De Diego Onsurbe, José Antonio

De Martino, Ivan

Degallaix, Jérôme

Del Sordo, Fabio

Desmond, Harry

Di Mauro, Mattia

Diaferio, Antonaldo

Dimitrijevic, Milan S.

Driggers, Jennifer $C$.

Dutil, Yvan

El Moumni, Hasan

Eldridge, Jan J.

Exter, Katrina

Fan, Junhui

Feng, James Q.

Flynn, Chris

Forbes, Duncan

Fortes, Elaine C. F. S.

Foschini, Luigi 
Foster, Adam

Frassino, Antonia M.

Fritschel, Peter K.

Fukazawa, Yasushi

Gasparrini, Dario

González, Gabriela

González, Marco Antonio Álvarez

Goyal, Arti

Guerrero, Martin A.

Gunther, Hans Moritz

Guo, Fulai

Hajduk, Marcin

Hajdukovic, Dragan

Hall, Evan

Hardcastle, Martin

Harko, Tiberiu

Harris, William

Hayashi, Kohei

Henry, Richard

Herdrich, Georg

Hernández, Xavier

Hillwig, Todd

Howarth, Ian

Huang, Zhiqi

Hull, Charles L. H. (Chat)

Hütten, Moritz

Izumi, Kiwamu

Izzard, Robert

Jones, Eriita

Karukes, Ekaterina

Kashi, Amit

Kastner, Joel

Kim, Dong-Woo

Koenigsberger, Gloria

Koivisto, Tomi

Koliopanos, Filippos

Komossa, Stefanie

Kovar, Jiri

Krishnendu, Naderi Varium

Krssak, Martin

Kumar, Korumilli Sravan

Kun, Emma

Kuns, Kevin

Kwok, Sun

Lalli, Roberto

Landoni, Marco

Langer, Norbert

Larionov, Valeri M.

Latter, William

Leonardi, Matteo

Liakos, Alexios
MacDonald, Nicholas

Malkov, Oleg

Manchado, Arturo

Manojlović, Lazo M.

Marmet, Louis

Marr, John

Marscher, Alan P.

Mastichiadis, Apostolos

Matsuno, Ken

McCuller, Lee

McDaniel, Alex

Mendigutía, Ignacio

Mennickent, Ronald

Micol, Benetti

Mielke, Eckehard

Migliori, Giulia

Mignani, Roberto

Miller, Hugh Richard

Mitra, Abhas

Mizuno, Yosuke

Monreal Ibero, Ana

Montez, Rodolfo

Morales, Juan Carlos

Motter, Juliana Cristina

Mueller, Guido

Munch, Jesper

Niarchos, Panagiotis George

Nilsson, Nils Albin

Nishikawa, Ken

Ntormousi, Evangelia

Nunes, Rafael C.

Odintsov, Sergei

Oliveira Mimoso, José Pedro

Otsuka, Masaaki

Ovgun, Ali

Panah, Behzad Eslam

Panotopoulos, Grigoris

Pardo, Kris

Pe'er, Asaf

Peimbert, Manuel

Perivolaropoulos, Leandros

Petri, Jerome

Piattella, Oliver

Pletser, Vladimir

Pradhan, Parthapratim

Pramanik, Souvik

Rahvar, Sohrab

Ramakrishnan, Venkatessh

Rechy-García, Jackeline S.

Rieger, Frank

Rincon, Angel 
Rodríguez, Mónica

Roshan, Mahmood

Rotondo, Marcello

Rubiera-Garcia, Diego

Sachkov, Mikhail

Sadun, Alberto C.

Salucci, Paolo

Sanchez Contreras, Carmen

Sánchez, Sebastian F.

Schlegel, Eric M.

Schneider, Christian

Schober, Jennifer

Schombert, James

Sharif, Muhammad

Sheykin, Anton A.

Sil'chenko, Olga

Silva, Laura

Sofue, Yoshiaki

Soker, Noam

Sokoloff, Dmitry Dmitrievich

Soloviev, Vladimir O.

Steinlechner, Sebastian

Stevens, Ian

Storm, Jesper

Taoso, Marco

Tarnopolski, Mariusz

Telada, Souichi

Theulé, Patrice
Toth, Viktor

Trigo-Rodríguez, Josep M.

Trushkin, Sergei

Trushkin, Sergey A.

Twu, Ruey-Ching

Ueta, Toshiya

Van De Steene, Griet C.

Vink, Jacco

Visser, Manus

Vladimir Ivanovich, Korchagin

Vlemmings, Wouter

Volk, Kevin

Webb, James R.

Wesson, Roger

Wienold, Martin

Wiita, Paul

Woodard, Richard

Wu, David

Wurm, Gerhard

Yousaf, Zeeshan

Yuan, Feng

Zacharias, Michael

Zasche, Petr

Zdziarski, Andrzej A.

Zhang, Jia

Zhou, Hongzhe

Zhuk, Alexander

Zijlstra, Albert 